\title{
31.1: Invited Paper: A Touching Story: A Personal Perspective on the History of Touch Interfaces Past and Future
}

\author{
Bill Buxton \\ Microsoft Research, One Microsoft Way, Redmond, WA, USA
}

\begin{abstract}
Touch screens have a 40+ year history. Multi-touch and some of the gestures associated with it, are over 25 years old. This paper aspires to provide some perspective on the roots of these technologies, and share some future-relevant insights from those experiences. Since the scope of the article does not permit a comprehensive survey, emphasis has been given to projects and insights that are relevant, but less-well known.
\end{abstract}

\section{Introduction}

The announcement of two new products in 2007, the Apple iPhone and Microsoft Surface, gave a serious boost to interest in touch interfaces - especially those that incorporate multi-touch. Since then, touch, multi-touch, and the gesture-based interfaces that they frequently employ, have become close to "must-have" features in several market segments, including mobile devices, desktop computers, laptops, and large format displays.

What is typically missed amongst this newfound interest - but also typical of virtually all "new" technologies - is how far back these techniques and technologies go [1][2]. For example, the use of touch input as a means to interact with computers began, at least, in the mid1960s, with early work being done by IBM [3] in Ottawa Canada[4], and the University of Illinois [5]. By the early 1970s, a number of different technologies had been disclosed.

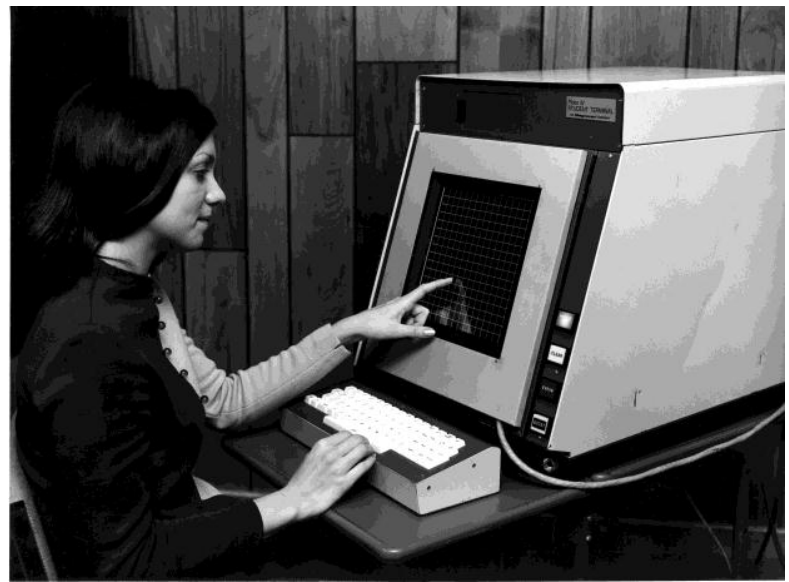

Figure 1: PLATO IV Terminal with touch screen and plasma panel display. (Courtesy of Archives of the University of Illinois, Urban Champaign. Found in RS: 39/2/20, Box COL 13, Folder COL 1313 Computer Ed. Research Lab / PLATO 1952-74)

By 1972, touch screens had left the labs and computer centers and entered selected grade-school classrooms as part ofthe PLATO IV system, illustrated in Figure 1. This was all the more remarkable when one considers that PLATO IV not only preceded the appearance of the personal computer and local area networks, its relatively wide deployment happened when Xerox PARC was just starting work on the Alto computer!

Through the 1970s-80s a number of different technologies were developed to support touch (such as capacitive, resistive, light interruption, and surface acoustic wave), and a number of different companies were founded to commercialize these technologies. Examples include, Elographics, Carroll Touch, and MicroTouch Systems.

As the options for the interface designer grew, so did the granularity of our understanding of the affordances of the available technologies and techniques. Nakatani and Rohrlich [6], for example, gave voice to the notion of "soft machines", what they defined as:

--- using the synergistic combination of real-time computer graphics to display "soft controls," and a touch screen to make soft controls operable like conventional hard controls.

However, as Gustave Flaubert said, "God is in the details," and getting the details wrong could make a good technology look really bad - as was the case with how cursor control was implemented on the early Apollo workstations, using an Elographics touchpad.

\section{Lost Along the Way}

From the time of PLATO IV to close to 2000, the use of touchsensing screens and tablets settled into a number or more-or-less niche markets. Touchpads/tablets (touch sensors not mounted directly over a display) became most visible on laptops, where they were (and are) the dominant technology used for cursor control. Touch screens were largely split into three main segments, kiosks (including ATM machines), point-of-sale devices (restaurants and retail, for example), and mobile devices (starting with PDAs, but as early as 1993 - as we shall discuss mobile phones).

Many of these markets were not very demanding in terms of the richness of the interaction techniques employed. Kiosks, for example, adopted mainly simple touch-to-select operations. At the same time, however, there was remarkable work which is not well-known, and hence worth highlighting.

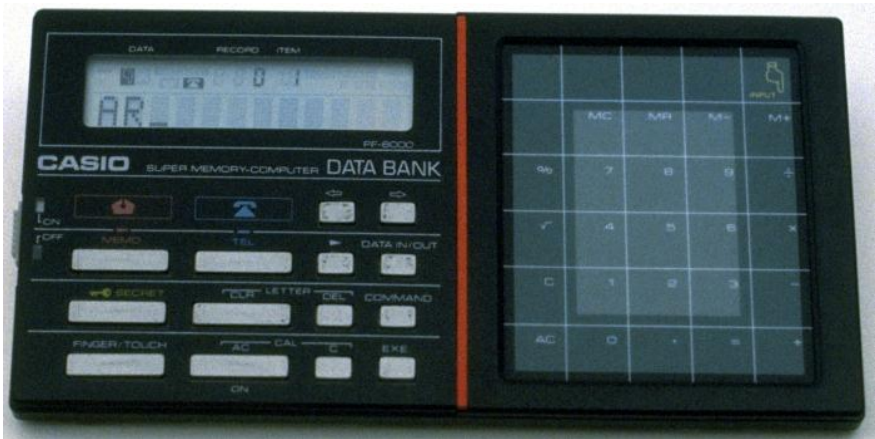

Figure 2: The PF-8000 Data Bank (1984). Characters can be entered by printing them on the touchpad with a finger. 
Take, for example, the Casio PF-8000, shown in Figure 2. This was a PDA that incorporated an address book and a calculator. It was released in 1984, which is when I got mine. As can be seen in the photo, the right side of the unit consists of a touchpad.

One of the ways that you could enter numbers was to tap them out on a virtual keyboard - defined by the white grid on the touchpad. More interesting, however, was the ability to enter alphanumeric information by tracing it out on the touchpad with your finger. You wrote each character on top of the previous one (segmentation was determined by the time interval between characters), so the whole touchpad surface was used for each character.

Lest one discount the relevance of this device because it used a touchpad, rather than touchscreen, in the same year, Casio released a calculator watch, the AT-550. The watch's crystal was a touch screen. Numbers and operators are "written" on the crystal, in the same manner as the PF- 8000 .

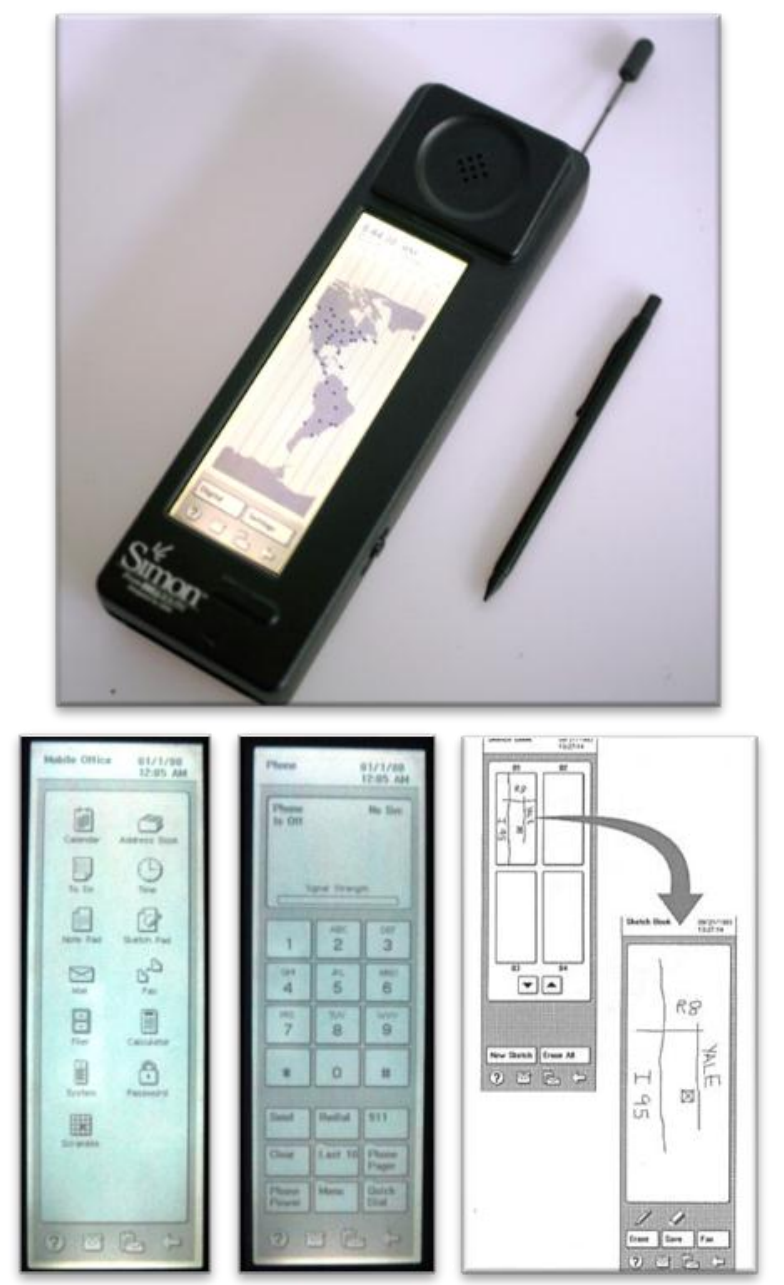

Figure 3: The Simon (1993). The phone's screen shows the display for setting the clock to world time. Interaction was via the touch screen, using either finger or stylus. L-R, the lower 3 images show (a) the desktop icons for accessing applications; (b) the phone dial pad; (c) the manual section for handling sketches and faxes. To place in context, there was no web browser: the World Wide Web had not yet happened yet!

Now flash forward and consider these devices in light of today's world of texting and TWITTER. A few minutes of experience with the PF- 8000 or the AT-550 make it clear that one can easily enter alphanumeric text without looking at the device. That is, the character recognition offers essentially the same eyes-free attribute that one has with touch typing on a QWERTY keyboard - something that I call "touch writing". Despite its relevance, this is something that is pretty much unavailable on any of today's mobile touch-entry devices. It is somewhat sobering to realize that Casio was able to do this in products commercially available 25 years ago - the same year that the very first Apple Macintosh computer was released!

Another important example is what I believe to be the world's first smartphone: the Simon [7], shown in Figure 3. This was developed jointly by IBM and Bell South, and first shown in 1993. How much this first smartphone anticipated the phones of today is only matched by how little it is known.

The Simon had only two physical controls: the on/off switch and the volume control. Everything else was controlled by the fullscreen touch display - which like the Palm Pilot (which appeared in 1996) - supported both finger and stylus control.

In addition to products, early innovative work was being undertaken in various research labs. Some of the most creative work is, likewise, little known. It was done by Chris Herot and Guy Weinzapfel at the Architecture Machine Group at MIT- the predecessor to MIT's Media Lab [8].

Their work is one of the first attempts to extend the range of touch sensing beyond just horizontal and vertical position. By mounting the touch-screen overlay on strain gauges, they were also able to sense vector information in six different dimensions, as illustrated in Figure 4: force in $\mathrm{x}, \mathrm{y}$, and $\mathrm{z}$, as well as torque about the $\mathrm{x}, \mathrm{y}$, and $\mathrm{z}$ axes.
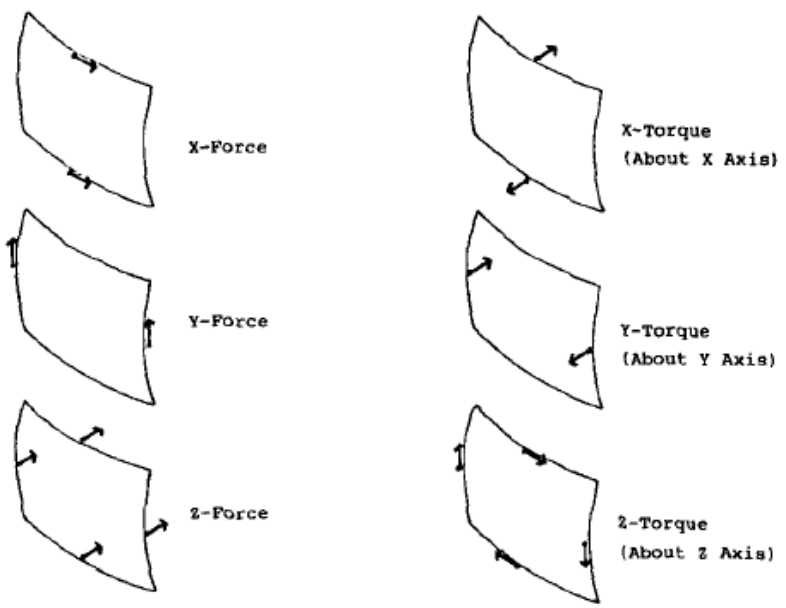

Figure 4: Multidimensional Touchscreen (1978): In addition to sensing position, this touchscreen [8] was capable of sensing 6 degrees of force vector information, including $x, y, z, x-$ torque, $y$-torque, and z-torque.

Of these additional dimensions, sensing force in $\mathrm{z}$ (pressure) is the only one that has gained any prominence, and even that is rare. But that speaks to the nature of the beast: the challenge is, the harder one pushes, the more friction there is in sliding the finger along the surface. Hence, there is an inherent conflict between forcevs. gesture articulation with touch interfaces. 
This is one area where sensing technology can make a difference. Capacitive sensing has a useful attribute in this regard, as was demonstrated by Buxton, Hill and Rowley [9], among others. It goes like this: if you push hard against a surface with your finger, the force tends to cause the fingertip to spread across the surface. Hence, there is a strong correlation between pressure and surface area. Furthermore, while capacitive technologies cannot sense pressure, per se, capacitance does vary with the area of contact. Hence, the technology can sense an approximation of pressure what I call "degree of touch". Knowing this means that the user can control the degree of touch by pressing lightly and varying the contact area. Thus, the user can assert degree of touch while avoiding the friction normally associated with pressure. Yet, just like pressure, this attribute is seldom exploited by interaction designers.

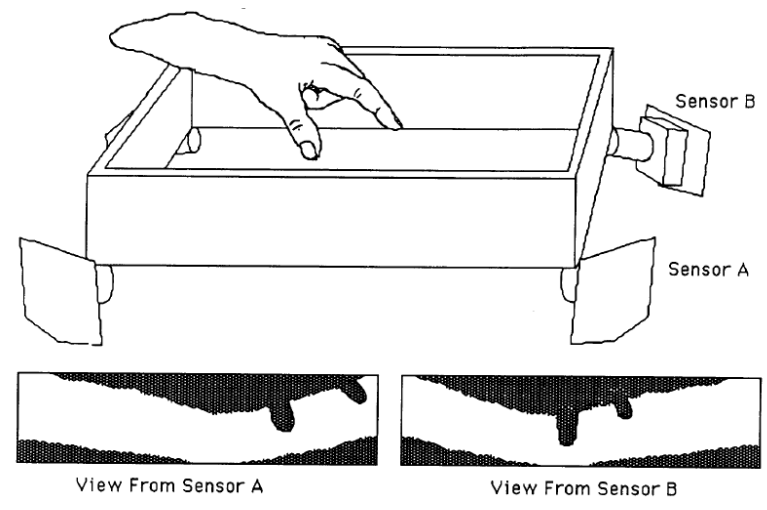

Figure 5: Sensor Frame: A prototype optical touch sensor that detects not only location, but also angle of approach[11].

In terms of exploring less commonly considered dimensions of touch sensing, I want to mention a novel approach to optical sensing of touch begun at Carnegie Mellon University by McAvinney [10], and developed further by Sensor Frame [11]. What they developed by 1988 was a device that used imaging across the display surface to sense touch location. However, unlike the light interruption techniques used with PLATO IV, this system - the Sensor Cube - used what were essentially cameras to detect the finger(s) in the volume above the display, rather than just at the display surface. Hence, as is illustrated in Figure 5, the angle of approach as well of the location of the finger could be determined.

\section{Multi-Touch}

The Sensor Cube had one other attribute that is sufficiently important to be worth a section on its own: the ability to sense simultaneously the location of multiple points of contact - multitouch. This also has a history.

In 1984 our group at the University of Toronto developed a capacitive multi-touch tablet capable of sensing degree of touch independently for multiple points of contact [12]. Our initial goal in this work was to make a digital hand drum - a musical percussion instrument. Since this was, I believe, the first multi-touch device reported in the peer-reviewed literature, it is often given credit for being the first multi-touch device. Such is not the case.

The roots of multi-touch lie partially in attempts to construct tactile sensors in robotics. Examples include Wolfeld [13] and Boie [14]. However, to the best of my research, the first use of multi-touch technology for manual control of a digital system was performed by Nimish Metha as part of his MSc thesis at the
University of Toronto [15]. This system has additional interest since it is the first use that I have found of capturing touch by using a video camera to optically capture shadows from the underside of a translucent surface - anticipating many current multi-touch systems, including Microsoft Surface. Just to emphasize this point, Metha's system was not only used to capture the shadows of fingers, but to capture and recognize shapes of objects as well!

However, to the best of my research, the first multi-touch display - the first sensor capable of simultaneously capturing multiple touch-points on a display - grew out of the aforementioned work on tactile sensors for robotics by Bob Boie.

After presenting our multi-touch tablet at SIGCHI in 1985, I was approached by Lloyd Nakatani of Bell Labs, Murray Hill, N.J. He invited me to visit the lab to see what they were doing. What I saw when I did so was a capacitive multi-touch screen that Boie had developed. Besides being transparent (ours was an opaque tablet), the performance of this device - in terms of response time - was far beyond what we had accomplished. Seeing the superiority of their system prompted me to stop working on the hardware part of the problem, and focus on the software. My assumption, hope, and expectation was that we would soon be able to get access to the Bell Labs technology. This turned out not to be the case, which was too bad, and the Bell Labs contribution went largely unknown in the larger community although it was openly shown to me, as well as others [16].

\section{A Sponge Without Water ...}

Thus far, the common factor in virtually all of the work discussed is a desire to extend the range of human capability that can be captured by touch technologies. The reality is that the simple poke-to-select techniques and soft keypads seen in early systems while useful - only scratched the surface of both the possible and the desirable.

One of the pioneers at really pushing the boundaries of capturing human gesture, and thereby laying the foundation for a great deal of current work, is Myron Krueger [17][18].

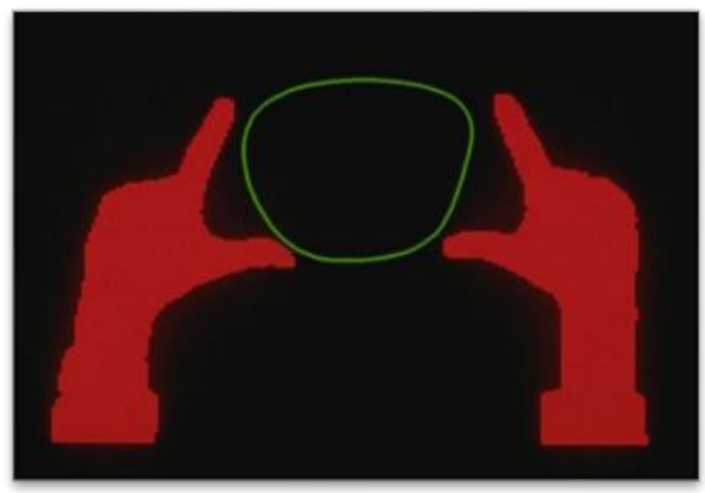

Figure 6: Myron Krueger's Pioneering VIDEODESK, early work using rich gestures. A two-handed pinch gesture is used to govern the shape of the closed object.

Myron's work was all about capturing human gesture, and demonstrating how it can be effectively used. He used a video camera to sense the current pose/action of the user, and then employed digital processing to isolate the human silhouette from the background. The silhouette was then analyzed and gestures extracted. These were then interpreted appropriately to bring 
about the intended response in the system. One such silhouette is illustrated in Figure 6. Here, two hands are used to control the shape of a closed object. The tips of all four extended fingers affect the shape - two from each hand in this case.

What is important to recognize in approaching Krueger's work is that the technology he used was secondary. It was a means to an end, not the end itself. The underlying point was all about the gesture, not the specifics of how it was captured. Hence, while his work did not sense touch, per se, it is relevant nevertheless.

The primary thing that does differentiate Krueger's work from touch systems is that contact with the physical device was not sensed. Hence, proximity, gesture, and/or dwell time - rather than physical contact - was required to initiate or terminate events. However major or minor one views the consequences of such differences, the fact remains that anyone practiced in the art of touch systems, and familiar with Krueger's work, was able to immediately adapt his work to this technology - and he explicitly wrote about its applicability to touch systems [18].

There is yet another class of gesture that has early roots, and which is also having significant impact on touch-based systems. It is that class of gestures where the resulting action is a function of both where one touches, and what direction(s) one strokes/moves, once having made contact. A common example of this found in many of today's mobile phones is the ability to move forward or backward from one image to another by touching the image and quickly sliding the finger left or right on the screen.

An early (1999) example of this technique was in a product called PortfolioWall [19], shown in Figure 7. What is important is that this gesture is a specific instance of a broader class of interaction techniques known generically as radial menus. Simply stated, radial menus characterize a class of interaction where the response to an action is a function of both where you touched, and the direction that you move in the gesture after that touch. The options used in viewing images using the PortfolioWall are shown in Figure 8.

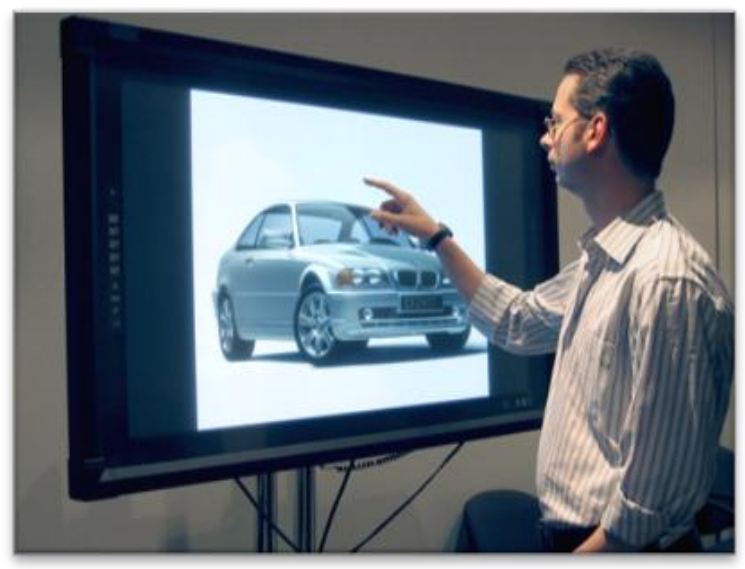

Figure 7: The PortfolioWall (1999). A sliding gesture to the left or right on top of the image moved to the next or previous image, respectively.

In addition to the left and right strokes, the radial menu shown supported the following gestures. A stroke up to the right enabled annotation, while a stroke down to the right enabled one to scale or crop the image. A stroke down closed the image and brought one back to the thumbnail view, while down to the left toggled between Play and Pause as a slideshow viewer. The menu was only displayed if one touched and held, without moving. Since the actions were easily learned, they were normally articulated without any graphical feedback - thereby illustrating the tight relationship between radial menus and their (in this case), associated eyes-free gestures.

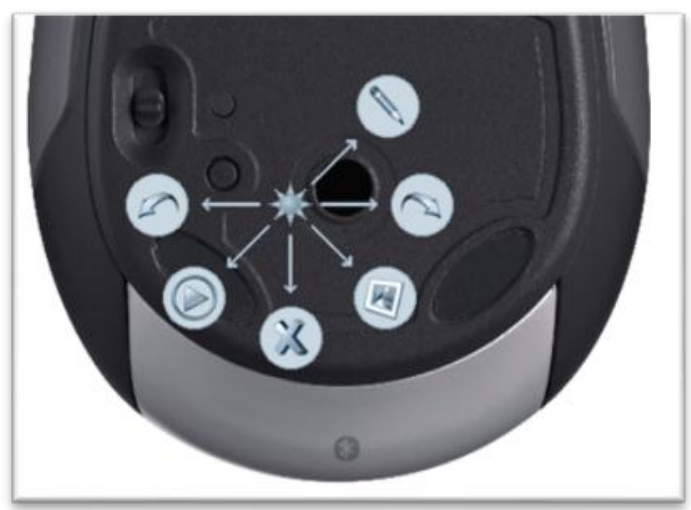

Figure 8: Radial Menu in PortfolioWall. The options when viewing a full sized image are shown by the menu.

Radial menus have a long history, beginning with the PIXIE system of Wiseman, Lemke and Hiles [20]. After a period of neglect, they were brought back into practice by Callahan, Hopkins, Weiser and Shneiderman [21], Hopkins [22], and Kurtenbach [23], for example. The key attribute that distinguishes them from conventional linear menus is that selection of action is determined by direction not distance. As human beings, we are not "wired" to make fine judgments of linear distance without looking. Yet, we are wired to be able to easily articulate gestures, eyes free, in any one of the eight primary and secondary directions of the compass. Therein lays the key to understanding that one should not think about radial menus as "just" menus. They also define a class of direction-based gestural interaction. And to emphasize this point, the work of Hopkins and Kurtenbach, cited above, and the PortfolioWall, makes clear that they work even if there is no menu displayed during their use.

The use of stroke direction to control the direction and type of scrolling on some current mobile devices (such as scrolling vertically, horizontally, or bi-dimensional dragging, depending on the direction of the stroke), is a good example of this, and demonstrates the relevance of radial menus to systems today.

\section{Moving Forward: Touch is a Means, Not an End}

Touch technologies are going to continue to evolve in terms of what they can sense and how they are used. Among other things, we are going to see ever more integration of the sensing technology with the display [24]. But while the technologies will continue to evolve, what must not get lost along the way is that it is just that, a technology, a means to an end. As I have discussed elsewhere [25], the conceptual model of the user interface is more important than the technology, and by that measure, two interfaces using different technologies (only one of which is touch) may have more in common than two where both do use touch.

Furthermore, while touch sensing can bring great value to an interface, even greater value can often be gained when it is used in combination, even simultaneously, with other technologies such as a stylus [26]. Again: everything is best for something and worst for something else. 


\section{Conclusions}

From beginnings such as these have emerged the touch technologies which are having such strong impact today these and a lot of outstanding work from a number of other researchers, designers and engineers whose work I had to neglect in this brief summary. Within this history lie important lessons and contributions that have the potential to inform our current decisions and thinking about these technologies, and their effective use going forward.

Finally, there is something in this history that can help shed light in our understanding of the nature of innovation. The length of time that it has taken for these technologies to reach "prime time" is the norm, not the exception. Innovation in our industry is almost always characterized by such a "long nose" - with $20+$ years being the norm [1][2]. Hence, this paper serves a second function as a reminder that the foundation of the next ten years of innovation were almost certainly planted over the past ten years, and are just waiting there to be cultivated.

\section{References}

[1] Computer Science and Telecommunications Board of the National Research Council, Innovation in Information Technology. (The National Academies Press, Washington DC, 2003).

[2] B. Buxton, "The Long Nose of Innovation." BusinessWeek.com (January 2nd, 2008). http://www.businessweek.com/innovate/content/jan2008/id2 008012_297369.htm

[3] P. Betts, C.J. Brown, J.J. Lynott, H.F. Martin, "Light Beam Matrix Input Terminal," IBM Technical Disclosure Journal, 9(5), 493-494, (1965).

[4] A.M. Hlady, "A Touch Sensitive X-Y Position Encoder for Computer Input," Proceedings of the AFIPS Fall Joint Computer Conference, Vol. 35, 545, (1969).

[5] F.A. Ebeling, R.S., Goldhor, R.L. Johnson, "A Scanned Infrared Light Beam Touch Entry System," Proceedings of Society of Information Displays," 134-135, (1972).

[6] L.H. Nakatani, J.A. Rohrlich, "Soft Machines: A Philosophy of User-Computer Interface Design," Proceedings of the ACM Conference on Human Factors in Computing Systems (CHI'83), 12-15, (1983).

[7] J.R. Lewis, "Reaping the benefits of modern usability evaluation: The Simon story," Advances in Applied Ergonomics: Proceedings of the 1st International Conference on Applied Ergonomics -- ICAE '96, 752-757, (1996).

[8] C. Herot, G. Weinzapfel, "One-Point Touch Input of Vector Information from Computer Displays," Computer Graphics, 12(3), 210-216, (1978).

[9] W. Buxton, R. Hill, P. Rowley, "Issues and Techniques in Touch-Sensitive Tablet Input," Computer Graphics, 19(3), 215-224, (1985).

[10] P. McAvinney, "The Sensor Frame - A Gesture-Based Device for the Manipulation of Graphic Objects," CarnegieMellon University, (1986).
[11] Sensor Frame, "The Sensor Frame Graphic Manipulator Final Report," NASA-CR-194243, (1992).http://ntrs.nasa.gov/archive/nasa/casi.ntrs.nasa.gov/19 940003261_1994003261.pdf.

[12] S.K. Lee, W. Buxton, K.C. Smith, "A multi-touch three dimensional touch-sensitive tablet," Proceedings of the ACM Conference on Human Factors in Computing Systems (CHI'85), 21-27, (1985).

[13] J.A. Wolfeld, "Real Time Control of a Robot Tactile Sensor," MSc Thesis. Philadelphia: Moore School of Electrical Engineering, (1981).

[14] R.A. Boie, "Capacitive Impedance Readout Tactile Image Sensor," Proceedings of the IEEE International Conference on Robotics, 370-378, (1984).

[15] N. Metha, "A Flexible Machine Interface," MSc. Thesis, Department of Electrical Engineering, University of Toronto, (1982).

[16] D.H. Rubine, "The Automatic Recognition of Gestures," MSc Thesis, Carnegie Mellon University, (1991).

[17] M.W. Krueger, Artificial Reality, (Addison-Wesley, Reading, MA, 1983).

[18] M.W. Krueger, T. Gionfriddo, K. Hinrichsen, "VIDEOPLACE - An Artificial Reality," Proceedings of the ACM Conference on Human Factors in Computing Systems (CHI'85), 35 - 40, (1985).

[19] W. Buxton, G. Fitzmaurice, R. Balakrishnan, G. Kurtenbach, "Large Displays in Automotive Design," IEEE Computer Graphics and Applications, 20(4), 68-75, (2000).

[20] N.E. Wiseman, H.U. Lemke, J.O. Hiles, "PIXIE: A New Approach to Graphical Man-Machine Communication," Proceedings of 1969 CAD Conference Southampton, IEEE Conference Publication 51, 463 - 471, (1969).

[21] J. Callahan, D. Hopkins, M. Weiser, B. Shneiderman, “An empirical comparison of pie vs. linear menus," Proceedings of the ACM Conference on Human Factors in Computing Systems (CHI'88), 95-100, (1988).

[22] D. Hopkins, "The Design and Implementation of Pie Menus,” Dr. Dobb's Journal, 16-26, (December 1991).

[23] G. Kurtenbach, "The Design and Evaluation of Marking Menus," Ph.D. Thesis, Department of Computer Science, University of Toronto, (1993).

[24] S. Izadi, S. Hodges, A. Butler, D. West, A. Rrustemi, W. Buxton, "ThinSight: A Thin Form-Factor Interactive Surface Technology," Communications of the ACM, 52(12), 90-98, (2009).

[25] B. Buxton, "The Mad Dash Towards Touch Technology," BusinessWeek.com, October 21, 2009. http://www.businessweek.com/innovate/content/oct2009/id2 0091021_629186.htm?chan=innovation_innovation $+\% 2 \mathrm{~B}+\mathrm{d}$ esign top+stories

[26] K. Hinckley, M. Pahud, B. Buxton, "Direct Display Interaction via Simultaneous Pen + Multi-touch Input," Society for Information Display (SID) Symposium Digest of Technical Papers, May 2010, Volume 41(1), Session 38, 537-540, (2010). 